\title{
THE CIRCULATION OF VECTOR FIELDS
}

\author{
VICTOR L. SHAPIRO ${ }^{1}$
}

1. Introduction. Operating in Euclidean three-space, $E^{3}$, with $u, v, w$ designating vectors and $e, t, n$ designating unit vectors, let $v(p)$ be a continuous vector field defined in the neighborhood of the point $p_{0}$. Also let $C_{n}\left(p_{0}, r\right)$ represent the circle with center $p_{0}$ and radius $r$, lying in the plane through $p_{0}$ normal to $n$, oriented by the usual right-hand rule. Define the upper circulation per unit area of $v$ at $p_{0}$ in the direction $n$, designated by $D_{n}^{*} v\left(p_{0}\right)$, as follows:

$$
D_{n}^{*} v\left(p_{0}\right)=\lim \sup _{r \rightarrow 0}\left(\pi r^{2}\right)^{-1} \int_{C_{n}\left(p_{0}, r\right)} v \cdot t d s,
$$

where $t$ designates the unit tangent and $d s$ the differential of arc length. Similarly define the lower circulation per unit area, $D_{* n} v\left(p_{0}\right)$, using lim inf. If $D_{* n} v\left(p_{0}\right)=D_{n}^{*} v\left(p_{0}\right)$ and both expressions are finite, designate this common value by $D_{n} v\left(p_{0}\right)$ and call it the circulation per unit area of $v$ at $p_{0}$ in the direction $n$.

The curl of $v$ is said to exist at the point $p_{0}$ if $D_{n} v\left(p_{0}\right)$ exists for every unit vector $n$ and if, furthermore, there exists a vector $w$ such that $w \cdot n=D_{n} v\left(p_{0}\right)$ for every unit vector $n, w$ is then called curl $v\left(p_{0}\right)$.

The curl of $v$ will be said to exist uniformly at $p_{0}$, if curl $v\left(p_{0}\right)$ exists and if, furthermore,

$$
\lim _{r \rightarrow 0}\left(\pi r^{2}\right)^{-1} \int_{C_{n}\left(p_{0}, r\right)} v \cdot t d s=n \cdot \operatorname{curl} v\left(p_{0}\right),
$$

uniformly in $n$.

It is clear that if $v(p)$ is in class $C^{1}$ in a neighborhood of the point $p_{0}$, then the curl of $v$ exists uniformly at the point $p_{0}$.

The above definitions are classical and can be found in most of the standard books on advanced calculus. In particular, pictorial illustrations of the above discussion can be found in $[1$, p. 351; 2, p. 278].

The following theorem will be proved in this paper:

THEOREM. Let $v(p)$ be a continuous vector field defined in an open set $R \subset E^{3}$. Suppose there exists three mutually orthogonal unit vectors $e_{1}, e_{2}, e_{3}$, and a constant $K$ such that $\left|D_{e_{j}}^{*} v(p)\right| \leqq K$ and $\left|D_{* e_{j} v} v(p)\right| \leqq K$ for $p$ in $R$ and $j=1,2,3$. Then curl $v$ exists uniformly almost everywhere in $R$.

Received by the editors July 16, 1962.

1 This research was supported by the Air Force Office of Scientific Research. 
The above theorem is the vector analogue of the classical theorem of Rademacher concerning Lipschitz functions and total differentials. (See [3, p. 310] or [6, p. 272].)

2. Proof of theorem. With no loss in generality, we can assume that $E^{3}$ has the usual Cartesian coordinate system with $p$ in $E^{3}$ given by $p=\left(x_{1}, x_{2}, x_{3}\right)$ and that $e_{j}$ is the unit vector $e_{j}$ in the direction of the $x_{j}$-axis, $j=1,2,3$. Furthermore, we can assume that $R$ is the interior of the unit ball in $E^{3}$. Then it follows from the proof in [5] (by reading $\sigma$ as a disc instead of a two-simplex) that with $K$ as in the hypothesis of the theorem that

$$
\left|\int_{C_{n}(p, r)} v \cdot t d s\right| \leqq 3 K \pi r^{2} \quad \text { if } C_{n}(p, r) \subset R .
$$

The first lemma we prove is the following:

LemMA 1. $D_{n}^{*} v(p)$ and $D_{*_{n}} v(p)$ are bounded (by $3 K$ ) Borel measurable functions in $R$ which are equal almost everywhere in $R$.

Let $A=\left\{p \mid D_{n}^{*} v(p)<a\right\}$, and let $B(p, r)$ be the closed three-ball with center $p$ and radius $r$. Fix $r_{1}$ with $0<r_{1}<1$, and let $k$ be an integer greater than $\left(1-r_{1}\right)^{-1}$. Then denote by $A^{k}$ the following set:

$$
\begin{aligned}
A^{k}=\left\{p \mid\left(\pi r^{2}\right)^{-1} \int_{C_{n}(p, r)} v \cdot t d s\right. & \\
& \left.\leqq a-k^{-1} \text { for } 0<r \leqq k^{-1} \text { and } p \text { in } B\left(0, r_{1}\right)\right\} .
\end{aligned}
$$

Since for fixed $r \leqq k^{-1},\left(\pi r^{2}\right)^{-1} \int_{C_{n}(p, r)} v \cdot t d s$ is a continuous function of $p$ in $B\left(0, r_{1}\right)$, we conclude that $A^{k}$ is a closed set. But then $A B\left(0, r_{1}\right)$ is an $F_{\sigma}$-set, and consequently $D_{n}^{*} v(p)$ is a Borel measurable function in $R$. A similar proof shows that $D_{*_{n}} v(p)$ is a Borel measurable function in $R$.

From (1), it follows that both $D_{*_{n} v}(p)$ and $D_{n}^{*} v(p)$ are bounded by $3 K$ in $R$. To show that $D_{*_{n} v} v(p)=D_{n}^{*} v(p)$ almost everywhere in $R$, set $u(p)=v(p)-[n \cdot v(p)] n$, and let $C_{n}\left(p_{0}, r_{1}\right) \subset R$. Then with $D_{n}\left(p_{0}, r_{1}\right)$, designating the closed disc having $C_{n}\left(p_{0}, r_{1}\right)$ as its boundary, we observe that $u(p)$ can be considered as a two-dimensional vector field defined on the two-dimensional set $D_{n}\left(p_{0}, r_{1}\right)$. Furthermore, it follows from (1) that for $p$ in $D_{n}\left(p_{0}, r_{1}\right), \lim \sup _{r \rightarrow 0}\left(\pi r^{2}\right)^{-1}\left|\int_{C_{n}(p, r)} u \cdot t d s\right| \leqq 3 K$. Consequently, by [4, Theorem 2], for almost every $p$ in $D_{n}\left(p_{0}, r_{1}\right)$,

(2) $\quad \lim \sup _{r \rightarrow 0}\left(\pi r^{2}\right)^{-1} \int_{C_{n}(p, r)} u \cdot t d s=\lim \inf _{r \rightarrow 0}\left(\pi r^{2}\right)^{-1} \int_{C_{n}(p, r)} u \cdot t d s$. 
But $D_{n}^{*} v(p)$ and $D_{*_{n}} v(p)$ are respectively the right and left sides of (2), and the conclusion of the lemma therefore follows from Fubini's theorem and the fact that $D_{n}^{*} v(p)$ and $D_{*_{n} v}(p)$ are Borel measurable functions in $R$.

Next, for $f(p)$ a Borel measurable function defined almost everywhere in $R$ which is, furthermore, essentially bounded on every compact subset of $R$, we define for $p$ in $B(0, r)$ where $0<r<1$ and $0<h<1-r$, the function $f_{h}(p)=\left(4 \pi h^{3} / 3\right)^{-1} \int_{B(0, h)} f(p+q) d q$. We say that $f(p)$ is mean-continuous at $p_{0}$ if $f(p)$ is defined at $p_{0}$ and if $f_{h}\left(p_{0}\right) \rightarrow f\left(p_{0}\right)$ as $h \rightarrow 0$. It is clear that for fixed $h, f_{h}(p)$ is continuous in $B(0, r)$. Furthermore, it is well known that if $f(p)$ is continuous in $R$, then $f_{h}(p)$ is in class $C^{1}$ in $B(0, r)$.

We next define for $p$ in $B(0, r)$, with $h$ and $r$ as above, the vector field $v_{h}(p)$ to be the vector field whose $e_{j}$ th component at $p$ is $\left[v \cdot e_{j}\right]_{h}(p), j=1,2,3$. It is clear that $v_{h}(p)$ is in class $C^{1}$ in $B(0, r)$. Also, the following lemma prevails.

Lemma 2. For $p_{0}$ in $B(0, r), D_{n} v_{h}\left(p_{0}\right)=\left[D_{n} v\right]_{h}\left(p_{0}\right)$.

We first observe that for $p$ in $B\left(0, r+r_{1}\right)$, where $r+r_{1}+h<1$, $v_{h}(p) \cdot t(p)=\left(4 \pi h^{3} / 3\right)^{-1} \int_{B(0, h)} v(p+q) \cdot t(p) d q$. Consequently,

$$
\begin{aligned}
& \int_{C_{n}\left(p_{0}, r_{1}\right)}{ }^{{ }^{\prime}}{ }_{h}(p) \cdot t(p) d s(p) \\
& \quad=\left(4 \pi h^{3} / 3\right)^{-1} \int_{B(0, h)}\left[\int_{C_{n}\left(p_{0}, r_{1}\right)} v(p+q) \cdot t(p) d s(p)\right] d q .
\end{aligned}
$$

Dividing both sides of (3) by $\pi r_{1}^{2}$ and passing to the limit as $r_{1} \rightarrow 0$, we obtain $D_{n} v_{h}\left(p_{0}\right)$ from the left side of (3). From (1), Lemma 1, and the Lebesgue bounded convergence theorem, we obtain $\left[D_{n} v\right]_{h}\left(p_{0}\right)$ from the right side of (3), and Lemma 2 is established.

By Lemma $1, D_{n} v(p)$ is a bounded Borel function defined almost everywhere in $R$. Set $Q_{n}=\left\{p \mid p\right.$ in $R$ and $D_{n} v$ is mean-continuous at $p\}$. By Lebesgue's theorem, $R-Q_{n}$ is a set of Lebesgue measure zero. The following lemma then holds:

Lemma 3. For $p_{0}$ in $Q_{e_{1}} Q_{e_{2}} Q_{e_{3}} Q_{n}$,

$$
D_{n} v\left(p_{0}\right)=\sum_{j=1}^{3} n \cdot e_{j} D_{e_{j}} v\left(p_{0}\right) \text {. }
$$

Since for $h$ small, $v_{h}(p)$ is in class $C^{1}$ in a neighborhood of $p_{0}$, $D_{n} v_{h}\left(p_{0}\right)=n \cdot \operatorname{curl} v_{h}\left(p_{0}\right)=\sum_{j=1}^{3} n \cdot e_{j} D_{e_{j}} v_{h}\left(p_{0}\right)$. But then by Lemma 2, 


$$
\left[D_{n} v\right]_{h}\left(p_{0}\right)=\sum_{j=1}^{3} n \cdot e_{j}\left[D_{e_{j}} v\right]_{h}\left(p_{0}\right)
$$

Using the fact that $p_{0}$ is in $Q_{e_{1}} Q_{e_{2}} Q_{e_{8}} Q_{n}$, (4) follows immediately from (5) on passing to the limit as $h \rightarrow 0$.

By a spherical lune, $\sigma$, we shall mean one of the four possible twodimensional closed sets determined by two great circles on a sphere. $|\sigma|$ will designate the two-dimensional area of $\sigma$, and $\partial \sigma$ will be oriented with respect to the outer normal of the sphere.

Lemma 4. Let $\sigma \subset \partial B(p, r)$ where $B(p, r) \subset R$. Then with $K$ as in the hypothesis of the theorem, $\left|\int_{\partial \sigma} v \cdot t d s\right| \leqq 3 K|\sigma|$.

Lemma 4 follows in the same manner as the analogous result was established for simplices in $[5$, p. 85]. We need only observe that on setting $Q_{1}=Q_{e_{1}} Q_{e_{2}} Q_{e_{3}}$, there exists a sequence of spherical lunes $\left\{\sigma_{j}\right\}_{j=1}^{\infty}$ contained in a small neighborhood of $\sigma$ with the following properties: (a) $\left|\sigma_{j}\right| \rightarrow|\sigma|$, (b) $\int_{\partial \sigma_{j}} v \cdot t d s \rightarrow \int_{\partial \sigma} v \cdot t d s$ and (c) the twodimensional measure of $Q_{1} \sigma_{j}$ on $\sigma_{j}$ is the same as $\left|\sigma_{j}\right|$.

Before proving the theorem, we note that if $n$ and $n_{0}$ are two unit vectors with $\left|n-n_{0}\right|<\epsilon, \epsilon>0$ and small, and if $\sigma_{1}$ and $\sigma_{2}$ are the two smaller spherical lunes determined by $C_{n}(p, r)$ and $C_{n_{0}}(p, r)$, then

$$
\left|\sigma_{k}\right| \leqq \epsilon \pi r^{2} \quad \text { for } k=1,2 .
$$

We now prove the theorem. Select a countable set $\left\{n_{j}\right\}_{j+1}^{\infty}$ of unit vectors with $n_{j}=e_{j}$ for $j=1,2,3$, which is dense in the set of all unit vectors, and set $Q=\prod_{j=1}^{\infty} Q_{n_{j}}$. Clearly, $R-Q$ is of Lebesgue measure zero.

Next, let $p$ be given in $Q$ and define $w(p)=\sum_{j=1}^{3} D_{e_{j}} v(p) e_{j}$. We propose to show that given $\epsilon>0$ and small, there exists $r_{0}$ (which depends on $p$ and $\epsilon$ ) such that if $0<r \leqq r_{0}$, then for every unit vector $n$

$$
\left|\left(\pi r^{2}\right)^{-1} \int_{C_{n}(p, r)} v \cdot t d s-n \cdot w(p)\right|<(9 K+1) \epsilon,
$$

where $K$ is the constant in the hypothesis of the theorem.

To do this, choose $M$ so large that $\left\{n_{j}\right\}_{j=1}^{M}$ constitutes an $\epsilon$-dense set. By Lemma 3 and the choice of $Q$, there exists an $r_{0}$ such that if $0<r \leqq r_{0}$,

$$
\left|\left(\pi r^{2}\right)^{-1} \int_{C_{n_{j}(p, r)}} v \cdot t d s-n_{j} \cdot w(p)\right|<\epsilon \quad \text { for } j=1, \cdots, M .
$$

Next, given a unit vector $n$, choose $n_{j}$ such that $\left|n-n_{j}\right|<\epsilon$. If 
$\sigma_{1}$ and $\sigma_{2}$ are the spherical lunes in (6) with $n_{j}$ playing the role of $n_{0}$, we then obtain from (6) and Lemma 4 , that for $0<r \leqq r_{0}$,

$$
\left|\int_{C_{n}(p, r)} v \cdot t d s-\int_{C_{n_{j}(p, r)}} v \cdot t d s\right| \leqq \sum_{k=1}^{2}\left|\int_{\partial_{\sigma_{k}}} v \cdot t d s\right| \leqq 6 K \pi r^{2} \epsilon .
$$

But then from (8), we see that for $0<r \leqq r_{0}$ the left side of $(7)$ is majorized by $(6 K+1) \epsilon+\left|\left(n-n_{j}\right) \cdot w(p)\right|$ which in turn is majorized by $(9 K+1) \epsilon$. The inequality in $(7)$ is consequently established, and the theorem is proved with $\operatorname{curl} v(p)=w(p)$.

In closing, we remark that if $S$ is a simple, oriented, $C^{1}$ surface contained in $R$ and $S-S Q$ is of measure zero with respect to the natural measure on $S$, it is not difficult to see from [4] and [5], that Stokes' theorem holds for $S$ with respect to $v$ and curl $v$.

\section{BIBLIOGRAPHY}

1. R. C. Buck, Advanced calculus, McGraw-Hill, New York, 1956.

2. W. Kaplan, Advanced calculus, Addison-Wesley, Cambridge, Mass., 1952.

3. S. Saks, Theory of the integral, Monographie matematyczne, Warsaw, 1937.

4. V. L. Shapiro, The divergence theorem for discontinuous vector fields, Ann. of Math. (2) 68 (1958), 604-624.

5. - Symmetric exterior differentiation and flat forms, Canad. J. Math. 14 (1962), 79-86.

6. H. Whitney, Geometric integration theory, Princeton Univ. Press, Princeton, N. J., 1957.

UNIVERSITY OF OREGON 